\title{
A novel polymorphism in exon 11 of the WKL1 gene, shows no association with schizophrenia
}

\author{
Andrew McQuillin ${ }^{1}$, Gursharan Kalsi ${ }^{1}$, Helen Moorey ${ }^{1}$, Graham Lamb ${ }^{1}$, Soraya Mayet ${ }^{1}$, \\ Digby Quested $^{2}$, Philip Baker ${ }^{1}$, David Curtis ${ }^{3}$ and Hugh Malcolm Gurling*,1
}

\begin{abstract}
${ }^{1}$ Molecular Psychiatry Laboratory, University College London, Department of Psychiatry \& Behavioural Sciences, Windeyer Institute of Medical Sciences, 46 Cleveland Street, London W1T 4JF, UK; ${ }^{2}$ Ealing Hospital, North West London Mental Health Trust, London UB1 3EU, UK; ${ }^{3}$ Department of Psychological Medicine, St Bartholomew's and Royal London School of Medicine and Dentistry, Whitechapel, London E1 1BB, UK
\end{abstract}

A missense mutation in exon 11 of the WKL1 gene on chromosome 22 was found to be associated with cases of catatonic schizophrenia in a single large pedigree. We have screened exon 11 of the WKL1 gene in $\mathbf{1 7 4}$ cases of schizophrenia, including cases of 22 cases of catatonic schizophrenia, but could not detect the previously reported mis-sense mutation. However in exon 11, we observed an insertion/deletion polymorphism, one-missense substitution and two synonymous substitutions. In addition, we also identified a nucleotide substitution in intron 11. All these polymorphisms appeared to be in complete linkage disequilibrium with one another. The polymorphisms were also identified in a UK pedigree with schizophrenia, however the polymorphisms did not segregate with the disease. To test for potential association between these polymorphisms and schizophrenia we sequenced an equal number of UK control individuals who were free of all psychiatric symptoms and had negative family histories for mental illness; the frequency of the insertion/deletion polymorphism was not significantly different in schizophrenia cases (42 out of 348 chromosomes, allele frequency $12 \%$ ) compared to normal controls (40 out of 356 chromosomes, allele frequency 11\%). The insertion/deletion was found to be in Hardy Weinberg equilibrium in both the schizophrenic and control groups. The insertion/deletion is composed of repeated sequence from exon 11 and intron 11 and is predicted to affect WKL1 protein structure. European Journal of Human Genetics (2002) 10, 491 - 494. doi:10.1038/sj.ejhg.5200837

Keywords: WKL1; MLC1; schizophrenia; catatonia; association; mutation

\section{Introduction}

A missense mutation in exon 11 of the WKL1 gene was first reported in an extended pedigree with seven cases of periodic catatonia and two obligate carriers of schizophrenia [MIM 181500]. ${ }^{1}$ This mutation was thought to be the cause of catatonic schizophrenia in this pedigree. WKL1 is thought to encode a brain expressed non-selective cation channel.

\footnotetext{
*Correspondence: Professor H Gurling, Molecular Psychiatry Laboratory University College London, Department of Psychiatry \& Behavioural Sciences, Windeyer Institute of Medical Sciences, 46 Cleveland Street, London W1T 4JF, UK. Tel: +44 (0) 2076799436 ;

Fax: +44 (0) 2076799437 ; E-mail: h.gurling@ucl.ac.uk

Received 7 February 2002; revised 17 April 2002; accepted 7 May 2002
}

The missense mutation, described by Meyer et al, ${ }^{1}$ was thought to disrupt a transmembrane domain of WKL1 and would therefore be likely to affect the function of the protein, possibly increasing susceptibility to schizophrenia. The WKL1 gene has also been investigated under an alternative gene name: MLC1. $^{2}$ Recessive mutations in the MLC1 gene are known to cause megalencephalic leukoencephalopathy with subcortical cysts (MLC [MIM 604004]). MLC patients have been identified with a number of different mutations in WKL1, however heterozygote carriers of these mutations did not exhibit psychiatric symptoms. Mutations in WKL1/MLC1 include a predicted splice variant of exon 11 that would result in the deletion of 17 amino acid residues of the exon, thought to form part of a transmembrane domain; 
and a substitution of $11 \mathrm{bp}$ with $3 \mathrm{bp}$ that resulted in a frameshift in the $5^{\prime}$ end of exon 11.

The burden of proof that a mutation causes a disease is high, and in the absence of any firm biochemical evidence from Meyer et al, ${ }^{1}$ that the mutation does indeed cause the disease, replication represents one of the most compelling means of obtaining additional evidence for cause and effect. Thus, whilst the WKL1 mutation was found only in a single pedigree with catatonic schizophrenia, it is thought that catatonic schizophrenia is probably closely related to all the common subtypes of schizophrenia ${ }^{3}$ and that from a familial perspective the different subtypes of schizophrenia are not sufficiently etiologically distinct syndromes that they can be investigated as distinct entities. $^{4}$

\section{Methods and materials}

Schizophrenic and control patients were ethnically selected to be of British or Irish ancestry. After complete description of the study to the subjects, written informed consent was obtained. All control subjects were screened for an absence of all mental disorders. Schizophrenics were diagnosed by a psychiatrist using the Lifetime Version of the Schizophrenia and Affective Disorders Schedule (SADS-L). ${ }^{5}$ The cases were also rated using the 90 -item OPCRIT checklist. ${ }^{6}$ Diagnoses were assigned using Research Diagnostic Criteria. ${ }^{7}$

Mutation screening was performed using the Li-Cor infrared fluorescent sequencing system. The method involved direct sequencing of PCR products amplified from genomic DNA. M13 tagged PCR primers that amplified the entire exon, and parts of the surrounding introns were designed. Primer sequences used were Intron 11 forward 5'-cacgacgttgtaaaacgacggagcgacttgaccactagc and Intron 10 reverse 5'-ggataacaatttcacacaggcacaggcttctcacctcct, where the sequence in italics corresponds to M13 sequences. Amplification products were $581 \mathrm{bp}$ in size. Amplification products were purified with Microclean reagent according to manufacturer's instructions (Microzone, UK). PCR products were then sequenced simultaneously in both directions with the SequiTherm Excel II DNA sequencing kit (Epicentre, USA) using two M13 primers fluorescently labelled with IRD700 and IRD800 (MWG Biotech, DE). Sequencing products were analysed on a Li-Cor 4200 automated sequencer (Li-Cor, USA). The samples were arranged

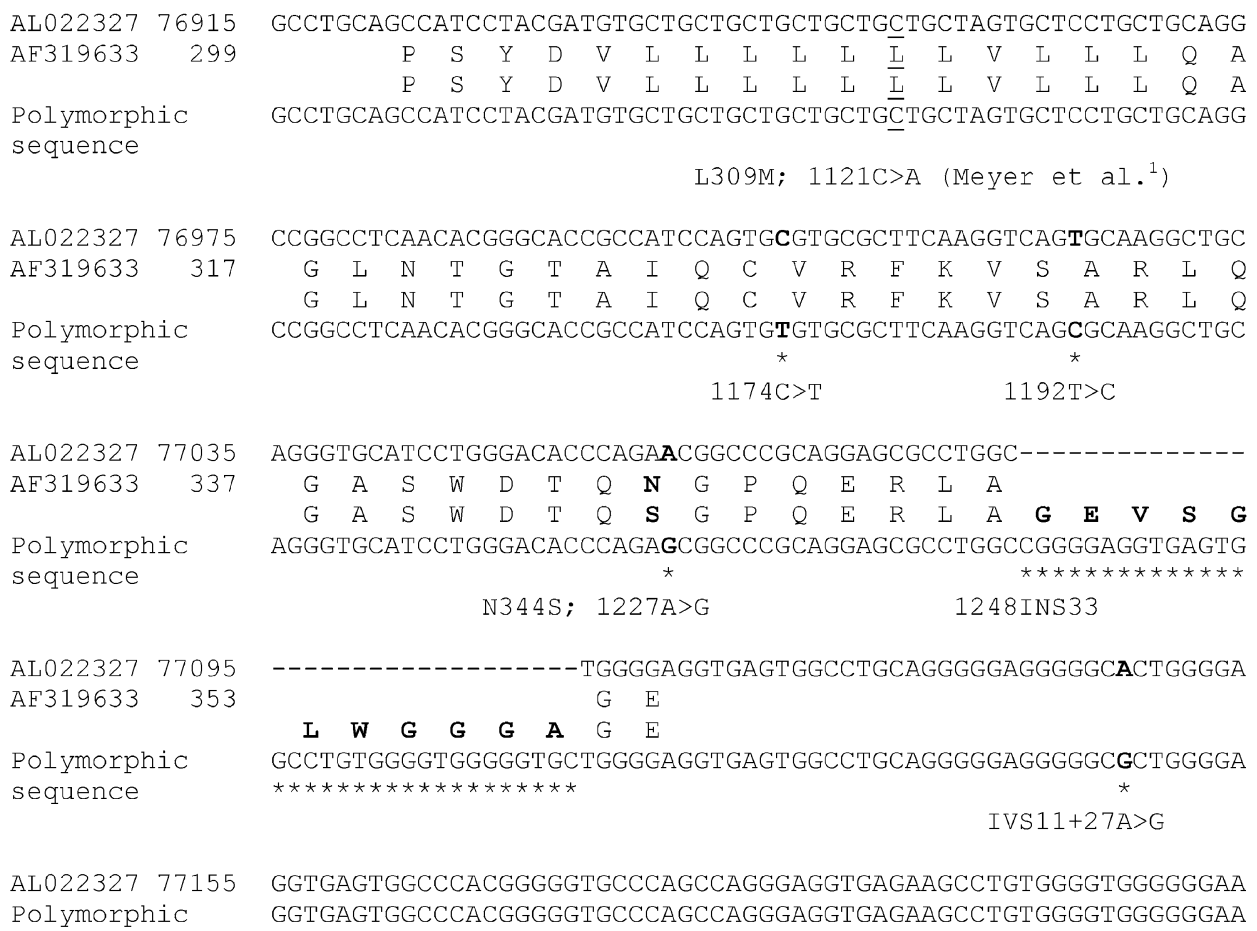

AL022327 77155 GGTGAGTGGCCCACGGGGGTGCCCAGCCAGGGAGGTGAGAAGCCTGTGGGGTGGGGGGAA Polymorphic GGTGAGTGGCCCACGGGGGTGCCCAGCCAGGGAGGTGAGAAGCCTGTGGGGTGGGGGGAA

Figure 1 Comparison of the nucleotide and deduced amino acid sequences of WKL1 (MLC1) exon 11 and surrounding intronic sequence. The upper nucleotide sequence (AL022327) is from the reverse complement of the published sequence (accession number AL022327). The upper amino acid sequence (AF319633) is from WKL1 and corresponds to the translation of WKL1 exon 11 (accession number AF319633). The lower nucleotide sequence represents the novel sequence described here (polymorphic). The deduced amino acid sequence from the novel sequence is also represented. Nucleotide substitutions or insertions are shown with an asterisk $\left({ }^{*}\right)$. The location of the missense substitution 1227A $>$ G leading to the amino acid substitution N344S is shown; along with synonymous substitutions $1174 \mathrm{C}>\mathrm{T}$ and $1192 \mathrm{~T}>\mathrm{C}$; and an intron 11 substitution IVS11+27A $>\mathrm{G}$. The position of the nucleotide substitution $1121 \mathrm{C}>\mathrm{A}$ leading to a L305M amino acid change reported by Meyer et al ${ }^{1}$ but not detected by us is shown (underlined). 
such that the guanosine dideoxy nucleotide terminated tracks from each of the samples were loaded together followed by the adenosine, thymidine and cytidine. This facilitated visual scoring of potential mutations and allowed recognition of polymorphisms in their heterozygote state. The validity of potential mutations was assessed by comparison with the gel image produced from simultaneous sequencing reactions primed from the opposite strand. The nucleotide sequence of selected samples was further clarified by analysis of these samples in the standard sequencing format. Alignment of nucleotide sequences in order to compare individuals was performed using AlignIR (LI-Cor, USA). An additional intron 11 reverse primer was designed that annealed $5^{\prime}$ to a repeat structure in intron 11. This primer paired with the original forward primer was also used to amplify and sequence sample individuals to further validate sequencing results. The sequence of this primer was 5 '-ggataacaatttcacacaggcaccccgtgggccactca.

\section{Results}

We sequenced exon 11 and its intron/exon boundaries in 174 UK schizophrenics, including cases of 22 cases of catatonic schizophrenia, and did not identify any individual that contained the mutation described by Meyer et al. ${ }^{1}$

However, we did observe a novel insertion/deletion polymorphism (with a predicted amino acid insertion of AGEVSGLWGGG 350-351), one-missense substitution (N344S) and two synonymous substitutions in exon 11 $(1174 \mathrm{C}>\mathrm{T}$ and $1192 \mathrm{~T}>\mathrm{C})$. In addition, we also identified a nucleotide substitution in intron 11 (IVS11+27A $>$ G (Table 1). All these polymorphisms were in complete linkage disequilibrium with one another and as such may be considered to form a haplotype. The insertion/deletion and other associated polymorphisms were identified in an affected member of a UK pedigree with schizophrenia. Other affected members of this pedigree were subsequently investigated, however the polymorphisms did not segregate with the disease.

We also sequenced an equal number of control individuals who were free of all psychiatric symptoms. The frequency of the insertion/deletion polymorphisms was not significantly different in cases and controls. Forty-two out of the $348(12 \%)$ chromosomes sequenced in schizo- phrenic patients had the insertion, whereas 40 out of 356 (11\%) of the chromosomes in control individuals possessed the insertion (Table 2). There was no evidence for genotypic association $\left(\chi^{2}=0.188,2\right.$ d.f. NS) or allelic association

Table 2 Genotype counts for the two alleles in schizophrenic and control samples

\begin{tabular}{|c|c|c|c|c|}
\hline \multirow[b]{2}{*}{ Genotype } & \multicolumn{3}{|c|}{ Schizophrenics } & \multirow[b]{2}{*}{ Controls } \\
\hline & Catatonia & $\begin{array}{l}\text { Never had } \\
\text { catatonia }\end{array}$ & $\begin{array}{l}\text { No data about } \\
\text { catatonia }\end{array}$ & \\
\hline 11 & 20 & 74 & 42 & 141 \\
\hline 12 & 2 & 24 & 8 & 34 \\
\hline 22 & 0 & 3 & 1 & 3 \\
\hline
\end{tabular}

Schizophrenic patients are also subdivided into those who had had catatonic symptoms, those who had never exhibited catatonic symptoms and those patients for whom we were unable to obtain data concerning catatonia. Allele 1 corresponds to the published sequence and allele 2 corresponds to the polymorphic insertion sequence.

Table 3 Results of chi-square analyses of genotype and allele counts for the insertion/deletion polymorphism in the schizophrenic and control samples

Association between WKL1 I/D polymorphisms in schizophrenics and in controls

$\begin{array}{ll}\text { Genotypic association } & \chi^{2}=0.188,2 \mathrm{df} p=0.9104 \\ \text { Allelic association } & \chi^{2}=0.119,1 \mathrm{df} p=0.7305\end{array}$

$\chi^{2}=0.119,1 \mathrm{df} p=0.7305$

Association between WKL1 I/D polymorphism in schizophrenics with catatonia and in schizophrenics without catatonia

Genotypic association $\quad \chi^{2}=3.229,2$ df $\mathrm{p}=0.1990$

Allelic association

$\chi^{2}=3.391,1$ df $\mathrm{p}=0.0655$

Association between WKL1 I/D polymorphism in schizophrenics with catatonia and in controls

Genotypic association

Allelic association

$\chi^{2}=1.793,2 \mathrm{df} p=0.4079$

$\chi^{2}=1.865,1$ df $p=0.1720$

Association between WKL1 I/D polymorphism in schizophrenics without catatonia and in controls

Genotypic association

$\chi^{2}=1.464,2$ df $\mathrm{p}=0.4810$

Allelic association

$\chi^{2}=1.536,1 \mathrm{df} p=0.2153$

The schizophrenic patients were also further subdivided into those who had experienced catatonic symptoms and those who had never experienced catatonic symptoms.

Table 1 Structure of the novel WKL1 exon 11 polymorphisms and the consequence if any, on amino acid sequence

\begin{tabular}{lll}
\hline Type of mutation & Nucleotide change & $\begin{array}{l}\text { Predicted effect on } \\
\text { amino acid sequence }\end{array}$ \\
\hline Missense substitution & $1227 \mathrm{~A}>\mathrm{G}$ & N344S \\
Insertion/deletion polymorphism & Insertion of 33 bases at 1248 & $\begin{array}{l}\text { Insertion of } 11 \text { amino acids at amino acid } 351 \\
\text { to 352 GEVSGLWGGGA }\end{array}$ \\
Synonymous substitution & CGGGGAGGTGAGGGCCTGTGGGTGGGGGTGC & No \\
Synonymous substitution & $1174 C>\mathrm{T}$ & None \\
Substitution in intron 11 & $1192 \mathrm{C}>\mathrm{C}$ & None \\
\hline
\end{tabular}

Nucleotide and amino acid numbers correspond to those used by Meyer et al. ${ }^{1}$ 
$\left(\chi^{2}=0.1191\right.$ d.f. NS) between the insertion/deletion and schizophrenia (see Table 3 ). In addition we were unable to identify genotypic $\left(\chi^{2}=3.2292\right.$ d.f. NS) or allelic association $\left(\chi^{2}=3.391 \quad 1\right.$ d.f. NS ( $P$ 0.065) between schizophrenic patients who had catatonic symptoms compared to those schizophrenics who were not recorded in the SADS-L/ RDC diagnostic system as having had catatonic symptoms (Table 3). Furthermore there was no evidence for either genotypic $\left(\chi^{2}=1.7932\right.$ d.f. NS) or allelic association $\left(\chi^{2}=1.8651\right.$ d.f. NS) in the comparison of the frequency of the insertion/deletion polymorphism between catatonic schizophrenics and controls (Table 3).

\section{Discussion}

WKL1 is thought to encode a non-selective cation channel expressed exclusively in brain. ${ }^{1}$ The protein is predicted to have eight transmembrane domains and one of these occurs in exon 11. ${ }^{1,2}$ Structural prediction analyses of the mutation found in the large pedigree with catatonic schizophrenia indicates that the presence of this missense mutation is likely to result in conformational changes of the mutant protein. ${ }^{1}$ Analysis of the polymorphisms described here using TMPred (http://www.ch.embnet.org/ software/TMPRED_form.html) and TOPred (http://bioweb. pasteur.fr/seqanal/interfaces/toppred.html) transmembrane domain prediction programmes indicate that the changes would not have a significant impact on the transmembrane domain encoded by exon 11.

We screened exon 11 in 174 UK schizophrenics and did not detect the substitution reported by Meyer et al. ${ }^{1}$ However we did identify a series of novel polymorphisms, including an insertion, which were in complete linkage disequilibrium with each other and which were present in an equal number of schizophrenic and control individuals. The identification of these polymorphisms in exon 11 of the same gene in healthy individuals suggests that this gene, or at least exon 11 WKL1, can withstand a number of changes without producing either the MLC phenotype or schizophrenia. However, it must be noted that megalen- cephalic leukodystrophy is a recessive disease, whereas schizophrenia is likely to contain both recessive and dominant subtypes that have both major gene and polygenic modes of transmission. It is still possible that the exon 11 MLC1/WKL1 base pair change found by Meyer et l $^{1}$ could cause a very rare autosomal dominant subtype of schizophrenia manifest as a catatonic schizophrenia but the evidence we have produced tends to favour the hypothesis that the chromosome 22 exon 11 mutation found by Meyer et $a{ }^{1}{ }^{1}$ is likely to be a neutral polymorphism unrelated to the aetiology of schizophrenia.

\section{Acknowledgements}

The research was funded by the Neuroscience Research Charitable Trust.

\section{References}

1 Meyer J, Huberth A, Ortega G et al: A missense mutation in a novel gene encoding a putative cation channel is associated with catatonic schizophrenia in a large pedigree. Mol Psychiatry 2001; 6: $302-306$.

2 Leegwater PA, Yuan BQ, van der Steen J et al: Mutations of MLC1 (KIAA0027), encoding a putative membrane protein, cause megalencephalic leukoencephalopathy with subcortical cysts. Am J Hum Genet 2001; 68: 831-838.

3 Tsuang MT, Winokur G: Criteria for subtyping schizophrenia. Clinical differentiation of hebephrenic and paranoid schizophrenia. Arch Gen Psychiatry 1974; 31: 43-47.

4 Kendler KS, McGuire M, Gruenberg AM, Walsh D: Outcome and family study of the subtypes of schizophrenia in the west of Ireland. Am J Psychiatry 1994; 151: 849-856.

5 Spitzer R, Endicott J, Robins E: Research Diagnostic Criteria for a selected group of functional disorders. 3rd edn. New York: New York State Psychiatric Institute, 1978.

6 McGuffin P, Farmer A, Harvey I: A polydiagnostic application of operational criteria in studies of psychotic illness - development and reliability of the Opcrit system. Arch Gen Psychiatr 1991; 48: $764-770$.

7 Spitzer R, Endicott J: The Schedule for Affective Disorders and Schizophrenia, Lifetime Version. 3rd edn. New York: New York State Psychiatric Institue, 1977. 